\section{Bendamustin plus Rituximab erfolgreich bei CLL}

\begin{abstract}
Mit Bendamustin und Rituximab wurden bei Patienten mit rezidivierender oder therapierefraktärer fortgeschrittener chronischer lymphozytischer Leukämie (CLL) bereits gute Ergebnisse erzielt. Nun wurde diese Kombination bei bisher unbehandelten CLL-Patienten untersucht.
\end{abstract}

E ür ur Patienten mit chronischer lymphozytischer Leukämie gibt es außer einer allogenen Stammzelltransplantation keine kurative Therapieoption. Für einen Großteil der Patienten kommt sie aber nicht infrage. In den vergangenen 50 Jahren wurden die Möglichkeiten der Chemotherapie bei CLL weiterentwickelt, sodass inzwischen hohe und dauerhafte Ansprechraten erreicht werden. Nachdem eine Chemoimmunotherapie mit Fludarabin/Cyclophosphamid und dem Anti-CD20-Antikörper Rituximab bzw. Bendamustin und Rituximab in einigen Studien bereits erfolgreich war, erfolgte nun eine weitere Studie mit letzterer Kombination bei bisher unbehandelten Patienten.

In die prospektive, nicht randomisierte Phase-II-Studie wurden 117 CLL-Patienten im Alter von 34 bis 78 Jahren auf- genommen. 46,2 \% der Patienten hatten ein Binet-Stadium C, $35 \%$ hatten eine Kreatinin-Clearance $\leq 70 \mathrm{ml} / \mathrm{min}$. und $25,6 \%$ der Studienteilnehmer waren mindestens 70 Jahre alt. Die First-LineTherapie erfolgte mit Bendamustin (90 $\mathrm{mg} / \mathrm{m}^{2}$ an den Tagen 1 und 2) und Rituximab $\left(375 \mathrm{mg} / \mathrm{m}^{2}\right.$ an Tag 0 des ersten Zyklus bzw. $500 \mathrm{mg} / \mathrm{m}^{2}$ an Tag 1 aller folgenden Zyklen). Insgesamt wurden bis zu sechs Zyklen verabreicht.

Die Gesamtansprechrate betrug $88,0 \%$, wobei das vollständige Ansprechen bei $23,1 \%$ und das partielle Ansprechen bei $64,9 \%$ lagen. In den verschiedenen genetischen Subgruppen gab es Unterschiede im Ansprechen. So betrug die Gesamtansprechrate $90 \%$ bei Patienten mit einer Deletion im Chromosom 11 (del(11q)), 94,7\% bei Patienten mit Trisomie 12 , und $89,4 \%$ bei $\mathrm{Pa}$ - tienten mit unmutierten IGHV-Genen (IGHV = variable Region der schweren Ketten von Immunglobulin). Patienten mit einer Deletion im Chromosom 17 $(\operatorname{del}(17 \mathrm{p}))$ hatten ein deutlich schlechteres Ansprechen von nur 37,5\%.

Nach einer medianen Beobachtungsdauer von 27 Monaten betrug das mediane ereignisfreie Überleben 33,9 Monate und das Gesamtüberleben 90,5\%.

Schwere Infektionen der Grade 3 oder 4 traten bei 7,7\% der Patienten auf. Neutropenien, Thrombozytopenien und Anämien der Grade 3 oder 4 waren bei 19,7, 22,2 bzw. 19,7\% der Patienten zu beobachten.

Fazit: Die Chemoimmunotherapie mit Bendamustin und Rituximab ist eine wirksame und sichere First-Line-Therapie für Patienten mit bisher unbehandelter CLL.

Judith Neumaier

Fischer K et al. Bendamustine in combination with rituximab for previously untreated patients with chronic lymphocytic leukemia: $A$ multicenter phase II trial of the German Chronic Lymphocytic Leukemia Study Group. J Clin Oncol. 2012;30(26):3209-16.

\title{
Lebenszeitgewinn für ältere Patienten mit AML?
}

\author{
Therapieoptionen für ältere Patienten mit akuter myeloischer Leukämie \\ (AML) sind begrenzt, v.a. bei eingeschränktem Allgemeinzustand und \\ Komorbiditäten. Eventuell tun sich allerdings bald neue Möglichkeiten auf.
}

— ine multizentrische, offene Phase-III- Studie verglich randomisiert Wirksamkeit und Sicherheit von Decitabin und Standardtherapien (nach Wahl von Patient und Behandler Supportivtherapie oder niedrig dosiertes Cytarabin) bei 485 Patienten über 65 Jahren (zwei Drittel waren über 70 Jahre alt) mit neu diagnostizierter AML, hohem oder mittlerem zytogenetischem Risiko und einem ECOG-Performancestatus von 0-2. Im Verum-Arm erhielten die Patienten 20 $\mathrm{mg} / \mathrm{m}^{2}$ Decitabin pro Tag als einstündige intravenöse Infusion an fünf aufeinanderfolgenden Tagen alle vier Wochen. Im Kontrollarm wurden sie nur supportiv oder mit $20 \mathrm{mg} / \mathrm{m}^{2} / \mathrm{d}$ Cytarabin s.c. an zehn aufeinanderfolgenden Tagen alle vier Wochen behandelt.
Bei der ersten Analyse nach dem Tod von 396 Studienteilnehmern (81,6\%) lag das Gesamtüberleben (OS) in der Decitabin-Gruppe nicht signifikant über dem im Kontrollarm (7,7 vs. 5,0 Monate; $\mathrm{p}=0,108$; Hazard ratio [HR] 0,85; 95\%-Konfidenzintervall [95\%-KI] 0,691,04). Eine nicht vorab geplante Auswertung nach dem Tod von 446 Patienten (92\%) bestätigte einen leichten Vorteil im OS mit einer HR von 0,82 (95\%-KI $0,68-0,99 ; p=0,037)$. Vollständige Remissionen mit (CR) und ohne Erholung der Plättchenzahl (CRp) waren mit $17,8 \%$ im Decitabin-Arm signifikant häufiger als im Kontrollarm mit 7,8 \% (Odds Ratio 2,5; 95\%-KI 1,4-4,8; p = 0,001). Die Rate unerwünschter Effekte war in beiden Armen vergleichbar, ob- wohl die Patienten im Decitabin-Arm median vier Zyklen erhielten, im Arm mit Wahlbehandlung dagegen nur zwei Zyklen. Am häufigsten traten bei Therapie mit Decitabin Thrombozytopenien und Neutropenien auf. Schwere Nebenwirkungen vom Grad 3/4 berichteten $59 \%$ der mit Decitabin behandelten und $55 \%$ der Patienten im Kontroll-Arm.

Fazit: Decitabin verbesserte bei älteren AML-Patienten die Ansprechraten gegenüber Standardtherapien, ohne dass es zu schwerwiegenden Toxizitäten kam. Die - allerdings ungeplante - späte OSAnalyse weist auf einen möglichen Vorteil von Decitabin auch beim Gesamtüberleben hin.

Friederike Klein

Kantarjian HM et al. Multicenter, randomized, open-label, phase III trial of decitabine versus patient choice, with physician advice, of either supportive care or low-dose cytarabine for the treatment of older patients with newly diagnosed acute myeloid leukemia. J Clin Oncol. 2012;30(21):2670-7. 